\title{
Preparation and Characterization of Mono- and Multilayers
}

\author{
Christoph BUBECK \\ Max-Planck-Institut für Polymerforschung, Postfach 3148, \\ D-6500 Mainz, Federal Republic of Germany
}

(Received November 26, 1990)

\begin{abstract}
A survey is given on recent developments in the preparation of ultrathin polymer films with the Langmuir-Blodgett technique. Low molecular weight amphiphiles and preformed polymers are compared with special emphasis on the structural requirements to stabilize the multilayers. It is shown how deviations from the traditional concepts of the LangmuirBlodgett technique can lead to improved film preparation for polymers. The characterization of the molecular orientation, especially of the molecular order of alkyl chains is obtained by means of infrared transmission and grazing incidence reflection spectroscopy. The lateral structure and morphology of multilayers can be characterized by a newly developed metal decoration technique. A cross-like network structure of silver decorated polyglutamate multilayers is resolved by polarization microscopy.

KEY WORDS Langmuir-Blodgett / Monolayers / Multilayers / structure / Orientation / Vibrational Spectroscopy / Surface Characterization / Polymers / polyoctadecylmethacrylate / Polyglutamate /
\end{abstract}

Monolayers and multilayers that are prepared by the Langmuir-Blodgett (LB) technique can be viewed as model systems to gain an improved understanding of membranes and interfacial phenomena of polymers. The field of organized monolayers and LB films has been growing during the last years and an increasing number of organic compounds have been designed to construct multilayer systems.' The manifold of low molecular weight amphiphiles and polymers can be arranged into several classes as shown schematically in Figure $1 .{ }^{2}$ Typical representatives of these classes of LB films will be described and compared with respect to their molecular order. The meaning of the molecular order and an ordered or disordered lateral structure is illustrated in Figure 2.

The characterization of the structure of these films is concerned with two questions. What is the influence of the molecular order in the range of the alkyl chains that are frequently used to gain the hydrophobic properties of the amphiphiles? What is the lateral microscopic structure of transfered films on solid substrates? 
a)

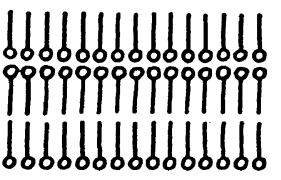

b) HHHHHHHH

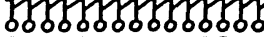

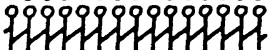

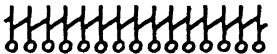


Figure 1. Comparison of the structure of Langmuir-Blodgett layers of different compounds (roughly simplified, view parallel to the layer plane):

a) classical amphiphiles,

b) reactive amphiphile after solid-state polymerization,

c) prepolymerized amphiphilic polymer,

d) rigid rod polymer substituted with flexible side chains.

The aim of this contribution is to elucidate how answers on these questions can be given by means of Fourier Transform Infrared (FTIR) spectroscopy under the conditions of Grazing Incidence Reflection (GIR) and polarization microscopy. The intrinsic birefringence of oriented LB films is usually too small for a direct image by polarization microscopy. Recently it was shown how the pleochroism of evaporated metal islands on LB films can be used to get information on the lateral structure of monolayers. This decoration technique will be described in the last chapter.

\section{EXPERIMENTAL}

The preparation of monolayers was performed on a Lauda model FW-1 Langmuir trough placed under a laminar flow hood. The monolayers were transfered on cleaned substrates like glass microscope slides, silicon wafers or gold covered glass mirrors under conditions as described recently. ${ }^{3-6}$ IR spectra of LB films were measured either in the transmission geometry using si-substrates or in GIR conditions. 5 , The evaporation of metals, polarization microscopy and electron microscopy were performed with commercial instrumentation. ${ }^{4,4}$

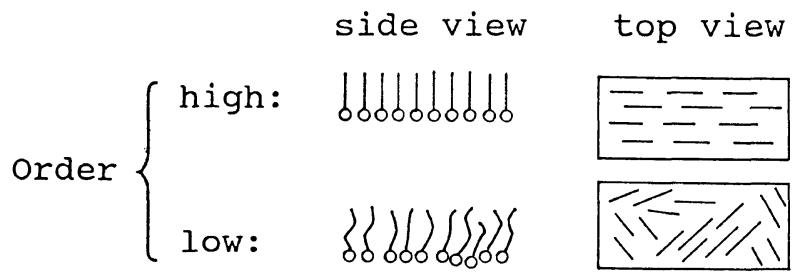

Figure 2. Scheme of high and low molecular order as seen in the side view. Ordered and disordered lateral structures are shown in a top view onto the plane of the substrate. 


\section{RESULTS AND DISCUSSION}

Amphiphiles of low molecular weight

structural investigations of the model compound Cdarachidate ( $\mathrm{Cd}-\mathrm{A})$ with GIR-FTIR spectroscopy have become very useful for an understanding of the basic properties of classical LB films. ${ }^{80}$ The transition dipole moments of the symmetric and antisymmetric stretching vibrations $v_{\mathrm{s}}(\mathrm{CH} 2)$ and $v a(\mathrm{CH} 2)$ are oriented perpendicular to the chain axis of $\mathrm{Cd}-\mathrm{A}$. If the alkyl chains of $\mathrm{Cd}-\mathrm{A}$ would be oriented perpendicular to the layer plane as indicated in Figure la, these vibrations could not be seen in the GIR experiment, because the resulting electric field vector of the incident IR radiation is normal to the layer plane. The experimental investigations of several groups show, that the intensity of the $v$ s,a $(\mathrm{CH} 2)$ bands ${ }_{4} \frac{i s}{8}-12$ comparable to the intensity of the $\nu_{\mathrm{s}, \mathrm{a}}(\mathrm{CH} 3)$ vibrations.

Therefore the intensity ratio of the $\mathrm{CH}_{2}-$ with respect to the $\mathrm{CH}_{3}-\mathrm{vibrations}$ can be used as a measure for the degree of order in the film. By use of perdeuterated $C d-A$ in distinct layer positions, the degree of order was investigated as a function of distance from the substrate surface. It was found that increased disorder or tilt of the whole molecular axis exists in the first monolayer on the substrate and the uppermost layers of the stack. In fact, it comes to an overlayer induced ordering of the layers underneath. These conclusions from vibrational spectroscopy are in good agreement with the evaluation of X-ray scattering experiments.

Consequently the high molecular order in the range of the alkyl chains is a prerequisite to obtain a reproducible monolayer transfer and reasonably good film properties of low molecular weight compounds.

The film stability of such amphiphiles is a severe problem, because melting of the crystalline packing of the alkyl chains and an irreversible loss of the periodic toyer structure is observed at temperatures higher than $100^{\circ} \mathrm{C}$.

\section{Polymerization in multilayers}

To improve the film stability, photopolymerization reactions in the multilayer have been widely applied as reviewed recently. ${ }^{14}$, The discussion is restricted to the example of polydiacetylenes, because the highly anisotropic absorption of the conjugated $\pi$-electron system of the polymer backbone can be used to gain images of the morphology of transferred monolayers. Polarization microscopic investigations clearly show uniquely oriented domains. ${ }^{14,16}$ The shape of the domains is elongated with respect to the transfer direction $\vec{t}$ of the substrate through the air-water interface, presumably due to shear forces during film compression and transfer. The orientation of the polymer chains changes between neighbouring domains.

Although the domain structure could be made visible in very few examples only, it is assumed that this is a general phenomenon in LB films that were prepared from low molecular weight amphiphiles. The occurence of domains is related to 
the crystallization properties of the alkyl chains. This leads to severe limitations for many applications due to light scattering and possible permeation at grain boundaries.

Preformed polymers with a flexible polymer backbone

As an example how preformed polymers can be used to built up LB films, some results of polyoctadecylmethacrylate (PODMA) are described. ${ }^{6}$ The structure of this polymer is shown in Figure 3 . At temperatures below $20^{\circ} \mathrm{C}$ a steep rise in the surface pressure/area $(\pi / A)$ isotherms can be seen. The transfer of the monolayer to solid substrates at $\pi=20 \mathrm{mN} / \mathrm{m}$ and $\mathrm{T}=17^{\circ} \mathrm{C}$ is possible but not very reproducible. As observed earlier the transfer ratio decreases at higher dipping cycles. However, if the transfer is performed at $\pi=$ $10 \mathrm{mN} / \mathrm{m}$ and $\mathrm{T}=28^{\circ} \mathrm{C}$, where a shoulder in the $\pi / \mathrm{A}$ isotherm indicates a liquid-analog phase, the transfer is y-type (that means a layer is transfered during the up- and down-trips). The transfer ratio stays constant up to at least 50 layers.

This leads to the conclusion, that the classical way to transfer low molecular weight amphiphiles being in the solid analog phase, is not applicable to polymeric monolayers. obviously the contribution of a liquid analogous phase is an advantage for good transfer properties of polymers.

The FTIR-GIR spectra of PODMA indicate that the molecular order in the range of the alkyl chains is reduced as compared to LB films of $\mathrm{Cd}-\mathrm{A}$. An orientation of the alkyl chains perpendicular to the layer plane is still visible to some degree, 6,18 as sketched in Figure $1 \mathrm{c}$.

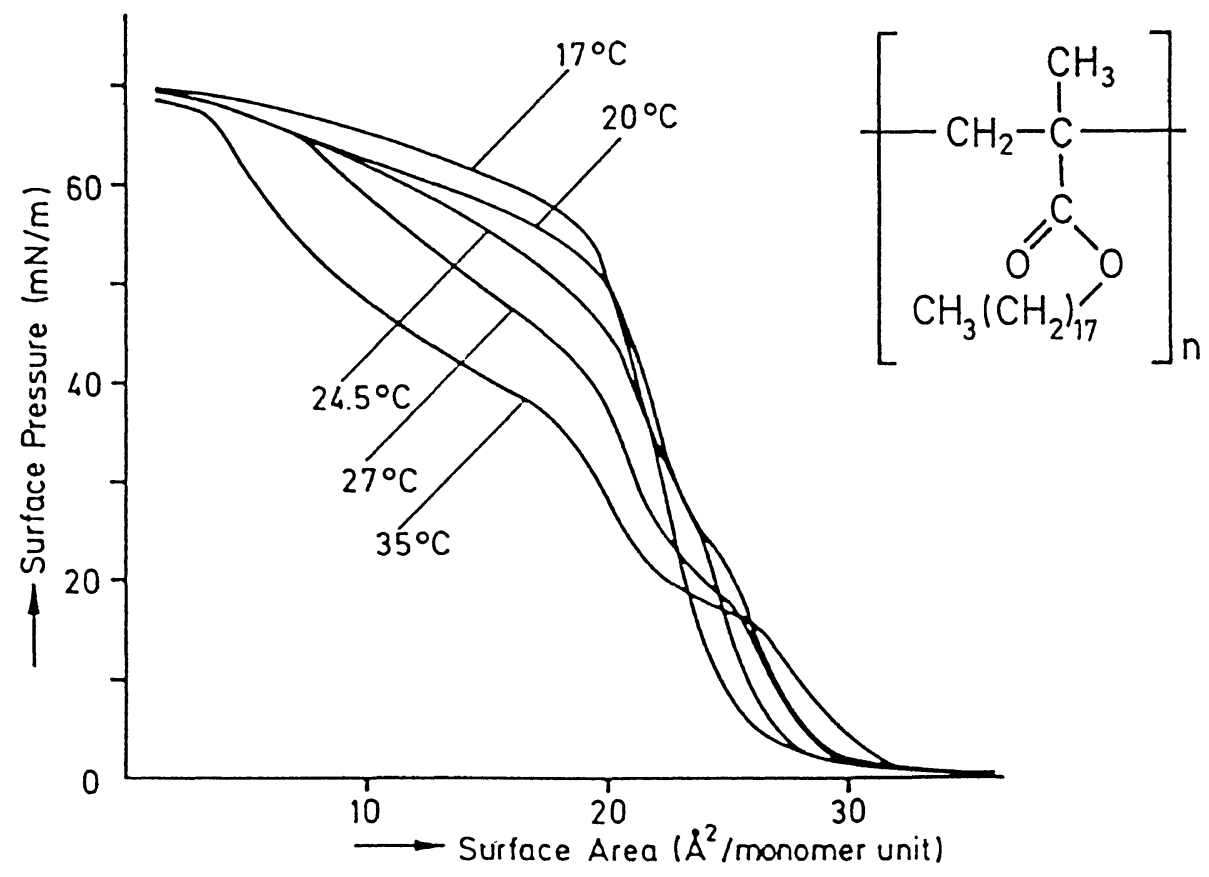

Figure 3. $\pi / A$ isotherms of isotactic PODMA at different temperatures (from Ref.6). 


\section{Rigid rod polymers with flexible side chains}

The use of rigid rod polymers that are substituted with alkyl chains in such a manner, that the alkyl chains cannot crystallize, has turned out to be a very promising concept for the preparation of LB films ${ }_{6,19,20}$ with improved transfer properties and film stability.6,19,20 Polyglutamates belong to this class of materials, because their polymer backbone can form a stiff $\alpha$-helix. Recently copolymers of polyglutamates with methyl and alkyl substitution were synthesized. The structure of poly-(r-methyl-L-glutamate-co$\gamma$-octadecyl-L-glutamate) abbreviated PG is:
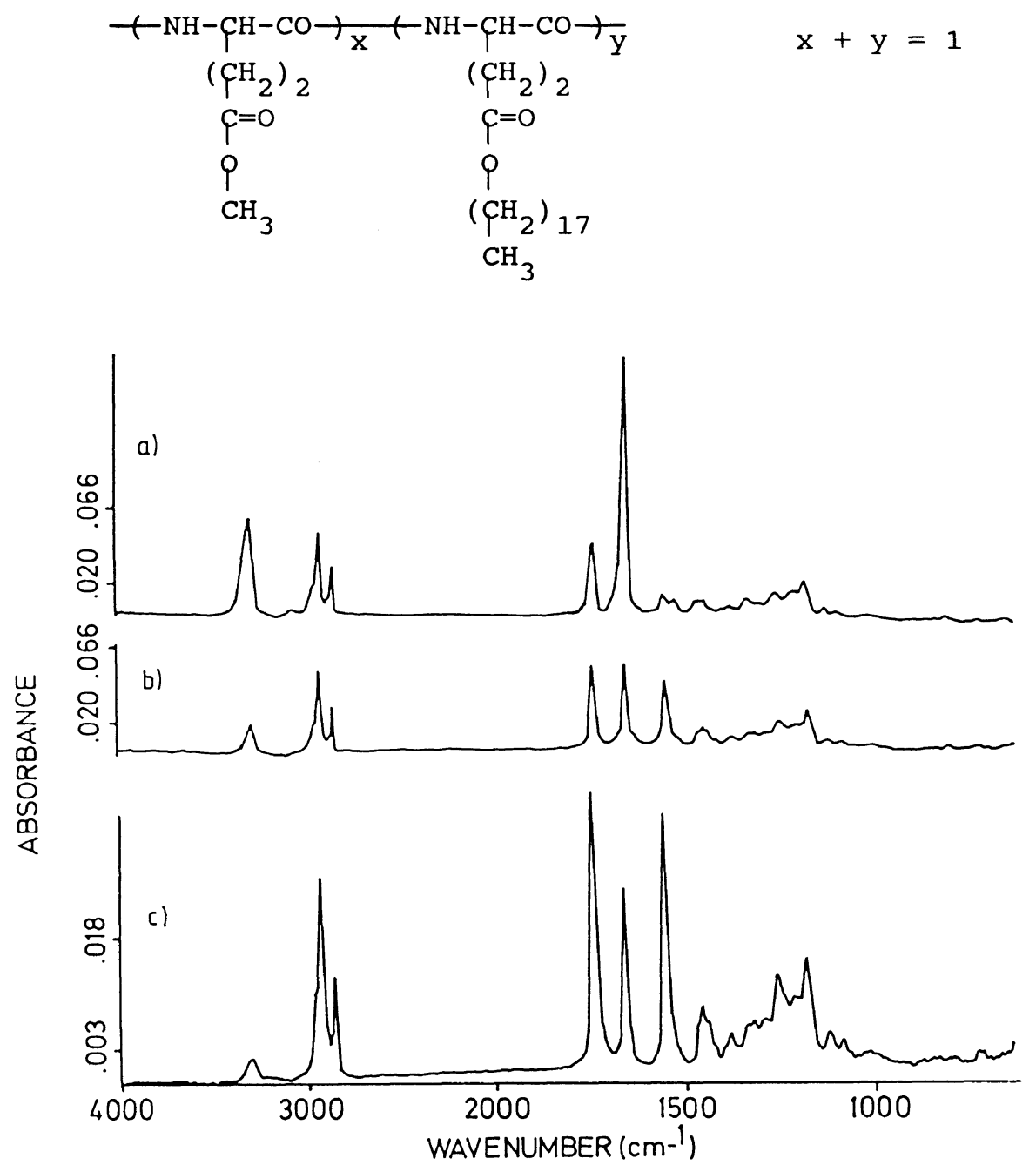

Figure 4. Infrared absorption spectra of LB multilayers of PG in different geometries:

a) transmission with polarized light parallel to $\vec{t}$,

b) transmission with polarized light perpendicular to $\vec{t}$,

c) grazing incidence reflection (GIR) with electric field vector $\vec{E}$ perpendicular to the $\mathrm{Au}$ mirror (from Ref. 6). 
Without substitution with long alkyl chains $(y=0)$, similar $\pi / A$ isotherms and transfer properties (z-type) are observed as reported earlier. ${ }^{2}$ At a degree of substitution of $y=0.2-0.4$, improved monolayer transfer is observed at $\pi$ $=20-25 \mathrm{mN} / \mathrm{m}$ and a subphase temperature of $20-25^{\circ} \mathrm{C}$. A y-type transfer with a constant transfer ratio of $100 \%$ is observed up to at least 200 layers. If $y$ is larger than 0.5 , the transfer ratio decreases at higher numbers of dippings.

The infrared absorption spectra of PG multilayers ( $y=$ 0.3 ) are shown in Figure 4. The shape of the GIR spectrum (c) in the range of the $\mathrm{CH}$ stretching vibrations clearly indicates that the alkyl chains are randomly oriented. This is the most striking difference to the $\mathrm{CH}$ vibration spectra of LB films from fatty acid salts. There a high molecular order in the packing of the alkyl chains was a prerequisite for the layer stabilization.

The spectra exhibit the typical amjde I(II) band at $1653 \mathrm{~cm}^{-1}$ and amide II(1) band at $1550 \mathrm{~cm}^{-1}$. This indicates that $P G$ is in the $\alpha$-helical conformation. The intensity ratios of the amide I and II bands in the polarized transmission spectra (a) and (b) show that the $\alpha$-helices of PG are oriented with their long axes preferably parallel to the transfer direction as observed earlier. ${ }^{22}$ Whether the statistical distribution of the $\alpha$-helices has only one maximum parallel to the transfer direction $\vec{t}$ or two maxima at angles $+\dot{\omega}$ and $-\bar{\omega}$ with respect to $\vec{t}$, can not be decided from the polarized absorption spectra alone.

\section{Surface characterization by metal decoration}

Recently it was found that the evaporation of noble metals like Ag and Au on top of LB films can be used $a_{3,4} \mathrm{a}$ In the initial stages of metal film growth, a nucleation to metal islands occurs as shown schematically in Figure 5. The sticking propability of metal atoms and their nucleation are extremely surface sensitive. Images of the decorated LB films can be obtained by direct visual inspection, because the metal islands have resonance absorption bands in the visible. Electron microscopy and polarization microscopy are further used to obtain the detailed structural information.

As an example, Figure 6 shows an electron microscopic image of Ag islands on 3 monolayers of Cd-A. The LB film was exposed to a silver flux corresponding to an average thickness of $50 \AA$ at the quartz crystal microbalance located near to the sample. A nucleation and coalescence of islands to chains with a statistically prefered orientation can be seen clearly. Obviously the anisotropic surface of the LB

Figure 5. Structural scheme of metal islands on a LB film.

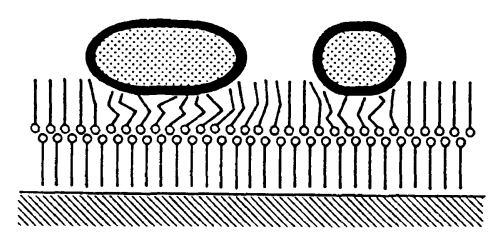


Figure 6. Electron microscopic image of a silver island film, deposited on top of 3 monolayers of $\mathrm{Cd}-\mathrm{A}$ (from Ref. 4).

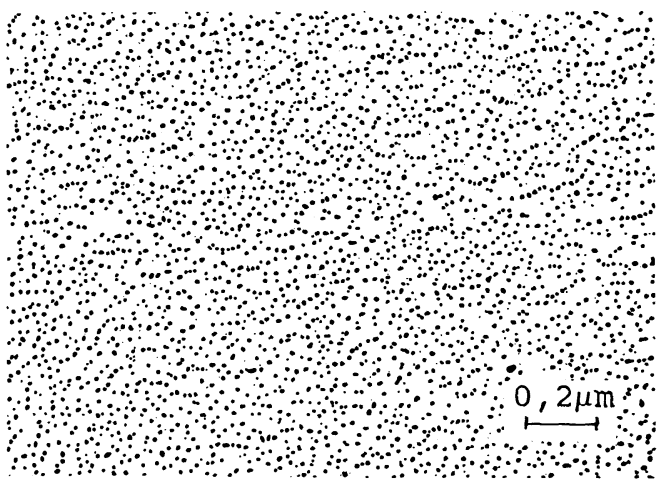

film leads to a distinct anisotropy of the mobility of the metal atoms, the distribution of nucleation sites and finally to the anisotropic coalescence of metal islands.

The optical properties of such oriented island clusters are mainly determined by dipolar coupling effects with a special importance of near field contributions, because the island sizes and their relative distances are all small as compared to the wavelength of light. Recent calculations show that two discrete absorption modes at frequencies $\omega x$ and $\omega_{y}$ exist. 23-25 If the external electric field $\vec{E}$ is oriented paraliel to the chain axis of the islands, called $\vec{x}$, then the mode $\omega x$ is exited. If $\overrightarrow{\mathrm{E}}$ is rotated by $90^{\circ}$, the mode $\omega_{y}$ is observed. The dipolar coupling between neighbouring islands leads to $\omega x<\omega_{y}$. Consequently such oriented island clusters have a strong pleochroism or manycolģredness. It can be seen in polarized absorption spectroscopy.

These strong polarization phenomena lead to the possibility to see the surface morphology of metal decorated LB films in the polarization microscope between crossed polarizers. The decoration technique is sensitive to the

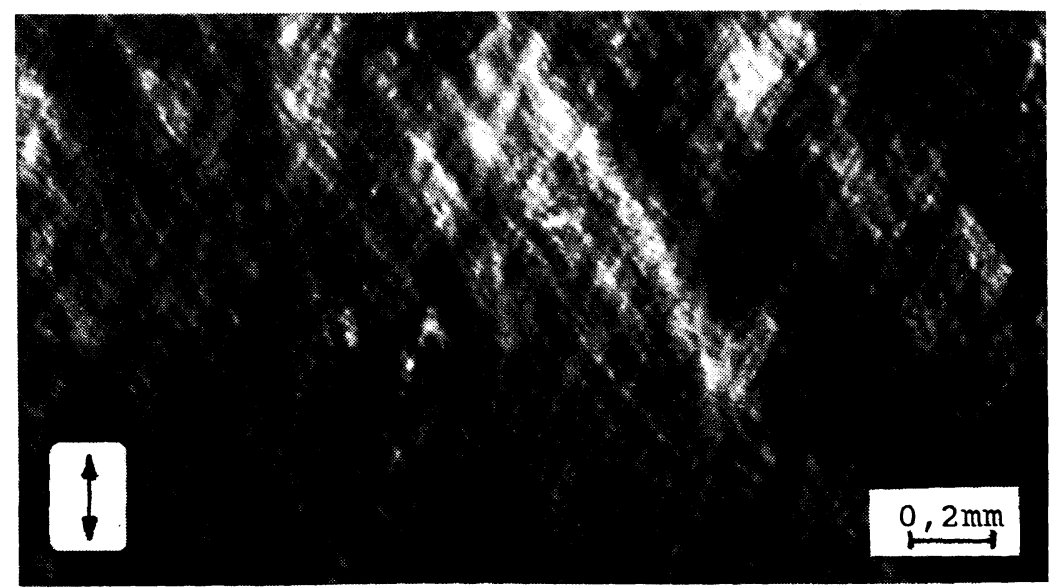

Figure 7. Optical microscopic image seen under crossed polarizers of a silver decorated LB film of polyglutamate. The $\vec{E}$ vector of the polarizer was oriented parallel to the transfer direction $\vec{t}$. 
structure of the uppermost monolayers as shown recently by the decoration of a step structure consisting of 2 monolayers of PG on top of 3 monolayers of Cd-A. The different morphologjes of these two classes of LB films were clearly resolved. A characteristic cross-like network structure of a Ag decorated LB film of PG is shown in Figure 7. Distinct axes are seen in the polarization microscope, that form angles of approximately $+35^{\circ} \pm 5^{\circ}$ and $-35^{\circ} \pm 5^{\circ}$ with respect to the transfer direction $\vec{t}$.

The origin of this cross-like network structure could be based on a statistically prefered orientation of the long axes of the $\alpha$-helices at these angles. This is a possibility that is compatibel with the dichroism observed in the FTIR spectra of Figure 4, but can not be prooven by them and needs further investigations.

The final conclusion is that the metal decoration method allows to characterize the surface morphology of oriented organic thin films by optical means and delivers a multitude of new information about the lateral structure of LB films that was not accessible before.

\section{ACKNOWLEDGEMENTS}

It is a great pleasure to acknowledge the contributions of the coworkers and the collaboration of T. Arndt, G. Duda, G. Lieser, A.J. Schouten and the stimulating interest of Prof. G. Wegner. Financial support for this work was given by the Bundesministerium für Forschung und Technologie under the project "ultrathin films of polymers".

\section{REFERENCES}

1. Proceedings of the 3. and 4. Int. Conf. on LB films, special issues Thin Solid Films, 159, 160 (1988), $178,179,180$ (1989).

2. C. Bubeck, D. Neher, A. Kaltbeitzel, G. Duda, T. Arndt, T. Sauer, G. Wegner, in J. Messier et al (Eds.)

"Nonlinear Optical Effects in Organic Polymers", Kluwer Acad. Publ. Dordrecht (1989) p.185.

3. C. Bubeck, Adv. Mater., 2, 537 (1990).

4. C. Bubeck, Thin Solid Films, 178, 483 (1989).

5. T. Arndt, C. Bubeck, Thin Solid Films, 159, 443 (1988).

6. G. Duda, A.J. Schouten, T. Arndt, G. Lieser, G.F. Schmidt, C. Bubeck, G. Wegner, Thin Solid Films, 159, 221 (1988).

7. R.G. Greenler, J. Chem. Phys., 44, 310 (1966).

8. D.L. Allara, J.D. Swalen, J. Phys. Chem., 86, 2700 (1982).

9. J.F. Rabolt, F.C. Burns, N.E. Schlotter, J.D. Swalen, J. Chem. Phys., 78, 946 (1983).

10. C. Naselli, J.F. Rabolt, J.D. Swalen, J. Chem. Phys., 82, 2136 (1985).

11. J. Umemura, T. Kamata, T. Kawai, T. Takenaka, J. Phys. Chem., 94, 62 (1990).

12. C. Bubeck, D. Holtkamp, Adv. Mater., in press (1991).

13. V. Skita, W. Richardson, M. Filipkowski, A. Garito, J.K. Blasie, J. Physique, 47, 1849 (1986). 
14. B. Tieke, Adv. Polymer Sci., 71, 79 (1985).

15. C. Bubeck, Thin Solid Films, 160, 1 (1988).

16. B. Tieke, G. Lieser, K. Weiss, Thin Solid Films, 99, 95 (1983).

17. S.J. Mumby, J.D. Swalen, J.F. Rabolt, Macromolecules, 19,1054 (1986).

18. T. Arndt, C. Bubeck, A.J. Schouten, G. Wegner, Mikrochim. Acta, II, 7 (1988).

19. E. Orthmann, G. Wegner, Angew. Chem. Int. Ed. Engl., 25, 1105 (1986).

20. T. Sauer, T. Arndt, D.N. Batchelder, A.A. Kalachev, G. Wegner, Thin Solid Films, 187, 357 (1990).

21. F. Takeda, M. Matsumoto, T. Takenaka, Y. Fujiyoshi, J. Colloid Interface Sci., 84, 220 (1981).

22. F. Takeda, M. Matsumoto, T. Takenaka, Y. Fujiyoshi, N. Uyeda, J. Colloid Interface Sci., 91, 267 (1983).

23. L.A. Ageev, V.K. Miloslavskii, I.N. Shklyarevskii, Opt.spectrosc., 40, 589 (1976).

24. J.M. Gerardy, M. Ausloos, Phys. Rev., B 22, 4950 (1980).

25. U. Kreibig in J. Davenas, P.M. Rabette (Eds.), "Contribution of Clusters physics to Materials science and Technology" NATO ASI Series E 104, 373 Martinus Nijhoff Publ., Dordrecht (1986). 